\title{
Asuntos de vida o muerte para el profesional en educación física
}

\author{
Luis Fernando Aragón-Vargas., Ph.D., FACSM \\ Escuela de Educación Física y Deportes, Universidad de Costa Rica \\ E-mail: luis.aragon@ucr.ac.cr \\ Manuscrito recibido: 18/06/08. Aceptado: 03/09/08
}

\begin{abstract}
RESUMEN
Aragón-Vargas, L. F. (2008). Algunos asuntos de vida o muerte para el profesional en educación física. Pensar en Movimiento: Revista de Ciencias del Ejercicio y la Salud, 6(1), 1-12. A pesar de que los profesionales universitarios en Educación Física reciben una formación bastante completa, es posible que desconozcan algunas condiciones o situaciones que pueden afectar la seguridad y hasta la vida misma de los participantes en eventos deportivos y recreativos que a menudo están bajo su supervisión y responsabilidad. Existe entonces un peligro real de que los profesionales mismos encargados de proteger al deportista sean quienes propicien accidentes y complicaciones, concretamente casos de rabdomiólisis por esfuerzo, casos de síndrome de células falciformes, accidentes por descargas eléctricas atmosféricas (rayería), y complicaciones de golpe de calor por esfuerzo al no enfriar rápida y efectivamente a la víctima.

El propósito de este estudio fue medir los conocimientos específicos en estos cuatro temas de interés. Se pasó una encuesta de 14 preguntas de selección única a 300 participantes en el XIV Simposio en Ciencias del Ejercicio y la Salud de la Universidad de Costa Rica (Octubre de 2007), escogidos por conveniencia entre aproximadamente 447 participantes inscritos, de los cuales $46 \%$ fueron profesionales graduados, $43 \%$ estudiantes de Educación Física, y 11\% que no reportaron su profesión u ocupación. Del total, 67\% son varones y 33\% mujeres. Se encontró que el nivel de conocimiento es muy limitado; en este manuscrito se da información clave sobre los cuatro temas, ya que el buen profesional en Educación Física debe conocer estas posibles complicaciones, de manera que pueda prevenirlas y así mejorar la seguridad y la experiencia de las personas a su cargo.
\end{abstract}

PALABRAS CLAVES: rabdomiólisis por esfuerzo, síndrome de células falciformes, rayería, golpe de calor por esfuerzo.

\section{ABSTRACT}

Aragón-Vargas, L. F. (2008). A few matters of life and death for the physical education professional. Pensar en Movimiento: Revista de Ciencias del Ejercicio y la Salud, 6(1), 1-12. Even though our Physical Education professionals undergo a rather comprehensive training, it may be possible for them to not be aware of several conditions or situations which may threaten the safety, and even the life of those participating in sporting or recreational events under their care. There is, therefore, a real danger that the very professionals responsible to protect the sports person may end up being the ones causing accidents and complications, e.g., exertional rhabdomyolysis, sickling, exertional heat stroke complications when a victim is not quickly and effectively cooled, and accidents from lightning.

The purpose of this study was to measure specific knowledge in these four areas of interest. A 14-item, multiple-choice survey was handed out to 300 participants in the University of Costa Rica's XIV Symposium on Exercise and Health Sciences (October, 2007), selected as a convenience sample from approximately 447 registered participants. $46 \%$ of them were professionals with university degrees, $43 \%$ were physical education students, and $11 \%$ did not report their current status. The sample was comprised of $67 \%$ males and 33\% females. Knowledge level was identified to be very limited; this paper includes key information on the four topics to help the good physical education professionals to be aware of these potential complications, improving safety and the general experience of those under their care. 
KEYWORDS: exertional rhabdomyolysis, sickle cell syndrome, lightning, exertional heat stroke.

A pesar de que los profesionales universitarios en Educación Física reciben una formación bastante completa, es posible que desconozcan algunas condiciones o situaciones que pueden afectar la seguridad y hasta la vida misma de los participantes en eventos deportivos y recreativos que a menudo están bajo su supervisión y responsabilidad. Por ejemplo, se han reportado casos de profesores de Educación Física y entrenadores que, sin intención, han provocado rabdomiólisis en sus pupilos (Clarkson, 1993; Clarkson, 2006). También se sabe que en algunas maratones de nivel mundial se utiliza equipo inadecuado para detectar la posibilidad de hipertermia en los corredores que colapsan, con el grave riesgo de un diagnóstico equivocado o tardío (Ronneberg, Roberts, McBean, \& Center, 2008). Existe entonces un peligro real de que los profesionales mismos encargados de proteger al deportista sean quienes propicien accidentes y complicaciones.

El objetivo de este estudio fue medir a priori, entre profesionales y estudiantes de Educación Física, los conocimientos específicos sobre cuatro condiciones potencialmente letales presentadas en un congreso científico, a saber: rabdomiólisis por esfuerzo, síndrome de células falciformes, accidentes por descargas eléctricas atmosféricas (rayería), y complicaciones de golpe de calor por esfuerzo. Se resumen además los cuatro temas y se dan recomendaciones para su prevención.

\section{METODOLOGÍA.}

Participantes. Se escogió una muestra por conveniencia de 300 personas, de los 447 participantes inscritos en el XIV Simposio de Ciencias del Ejercicio y la Salud, organizado por la Escuela de Educación Física y Deportes de la Universidad de Costa Rica en Octubre de 2007. El grupo estuvo conformado por $46 \%$ de profesionales graduados, $43 \%$ estudiantes de educación física, y $11 \%$ que no reportaron su profesión u ocupación. Del total, 67\% son varones y 33\% mujeres.

Instrumentos. Se preparó una encuesta con catorce preguntas de selección única (Anexo 1).
Cuatro preguntas estaban relacionadas con la rabdomiólisis, una con el síndrome de células falciformes, dos con las descargas eléctricas atmosféricas, y tres preguntas con la detección y manejo del golpe de calor por esfuerzo. Se incluyeron cinco preguntas adicionales en el cuestionario relacionadas con temas de manejo común de los educadores físicos. El cuestionario tiene validez aparente; en la discusión se respalda la escogencia de la respuesta correcta a cada pregunta.

Procedimientos. Los 300 cuestionarios se entregaron individualmente a las personas seleccionadas, el día de la inscripción en el Simposio ya mencionado. Se recogieron 168 documentos debidamente contestados por los participantes, previamente a la presentación del tema por el expositor.

Análisis de datos. Las respuestas a la encuesta fueron tabuladas, y se reportan como porcentajes del total de cada pregunta.

\section{RESULTADOS.}

La distribución porcentual de las respuestas a las preguntas relacionadas con rabdomiólisis se presenta en la Figura 1. La mayoría de los encuestados opina que el dolor muscular retardado es causado por la acumulación de ácido láctico, que la creatina fosfokinasa se eleva cuando hay acidosis excesiva durante el ejercicio intenso, y que la mioglobina transporta el oxígeno en la sangre.

En lo que respecta al peligro de complicaciones por rabdomiólisis o por síndrome de células falciformes, el $22 \%$ de los encuestados reconoce que el educador físico nunca debe utilizar el ejercicio como castigo porque existen casos de estudiantes que han muerto por esta causa. La mayoría opina que el problema radica en el generar en los alumnos una actitud negativa hacia el ejercicio. 

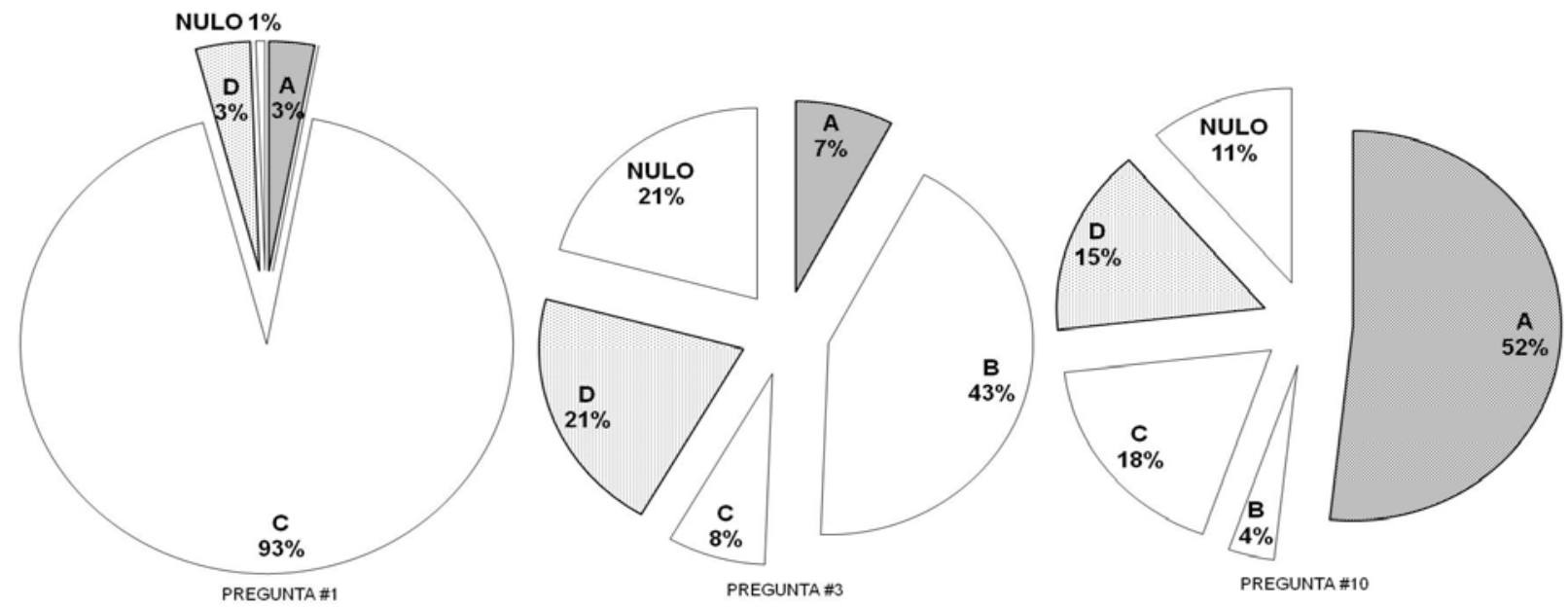

Figura 1. Conocimiento general sobre rabdomiólisis. Pregunta \#1: El dolor muscular retardado (A) Es algo pasajero que siempre se resuelve solo, con el tiempo, (B) Es potencialmente mortal, (C) Es causado por la acumulación de ácido láctico, (D) Es provocado por contracciones concéntricas. Pregunta \#3: Aparte de indicar que podría haber habido un infarto al miocardio, el CPK se eleva (A) Después de nadar en agua muy fría, (B) Cuando hay acidosis excesiva durante el ejercicio intenso, (C) Cuando ha ocurrido rabdomiólisis, (D) Por deshidratación aguda. Pregunta \#10: La mioglobina (A) Transporta el oxígeno en la sangre, (B) Puede provocar una falla renal si su concentración en sangre sube mucho, (C) Está relacionada con la potencia y la capacidad anaeróbica muscular, (D) Es una hormona regulada por el metabolismo aeróbico.

La Figura 2 muestra la distribución porcentual de las respuestas a las preguntas relacionadas con el peligro de accidentes por descargas eléctricas. Un $47.6 \%$ de los encuestados sugirió suspender las actividades al aire libre y buscar refugio cuando hay una tormenta eléctrica cerca, y $40.5 \%$ indicó que en Costa Rica mueren anualmente alrededor de 7 personas por estos accidentes.

La Figura 3 muestra la distribución porcentual de las respuestas a las preguntas sobre manejo de complicaciones serias por calor. La mayoría de los encuestados opina que cuando un atleta sufre un golpe de calor, se le debe enfriar primero y transportar después; la mayoría opina que la manera más efectiva y segura de enfriarlo es rociándolo repetidamente con agua fresca. El método más favorecido para confirmar la presencia de hipertermia es tocar la frente con el dorso de la mano.
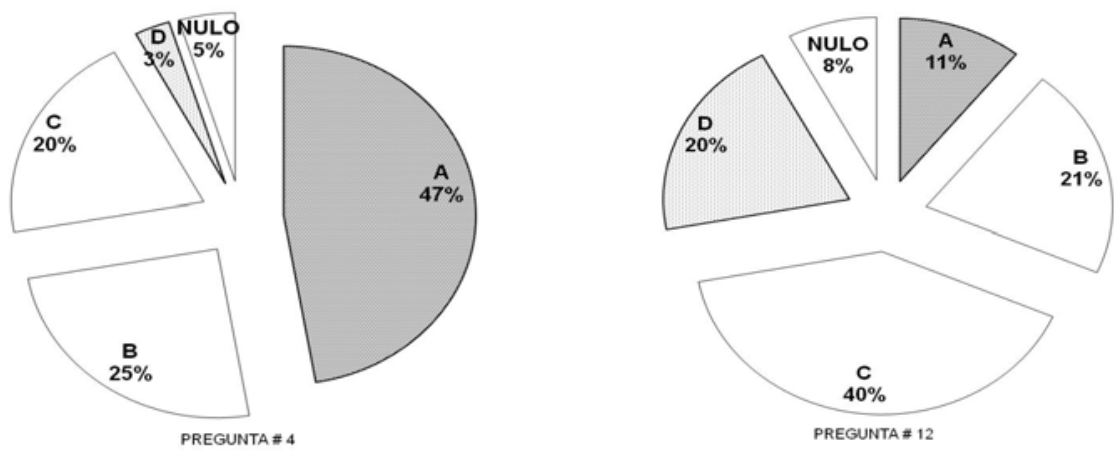

Figura 2. Conocimiento sobre el peligro de accidentes por descargas eléctricas (rayos). Pregunta \#4: Cuando hay una tormenta eléctrica a 5 kilómetros de una cancha abierta de fútbol, es necesario suspender el juego o entrenamiento y buscar refugio apropiado (A) y esperar 30 minutos después de la caída del último rayo, (B) sólo si los rayos tienen una frecuencia muy alta, (C) sólo si parece que la tormenta se acerca, (D) no es necesario suspender el juego. Pregunta \#12: En Costa Rica muere un número importante de personas por descargas eléctricas (rayería) al año mientras trabajan, se recrean, 0 practican deporte al aire libre, a saber (A) 2 personas por año, (B) 4 personas por año, (C) 7 personas por año, (D) 20 personas por año. 

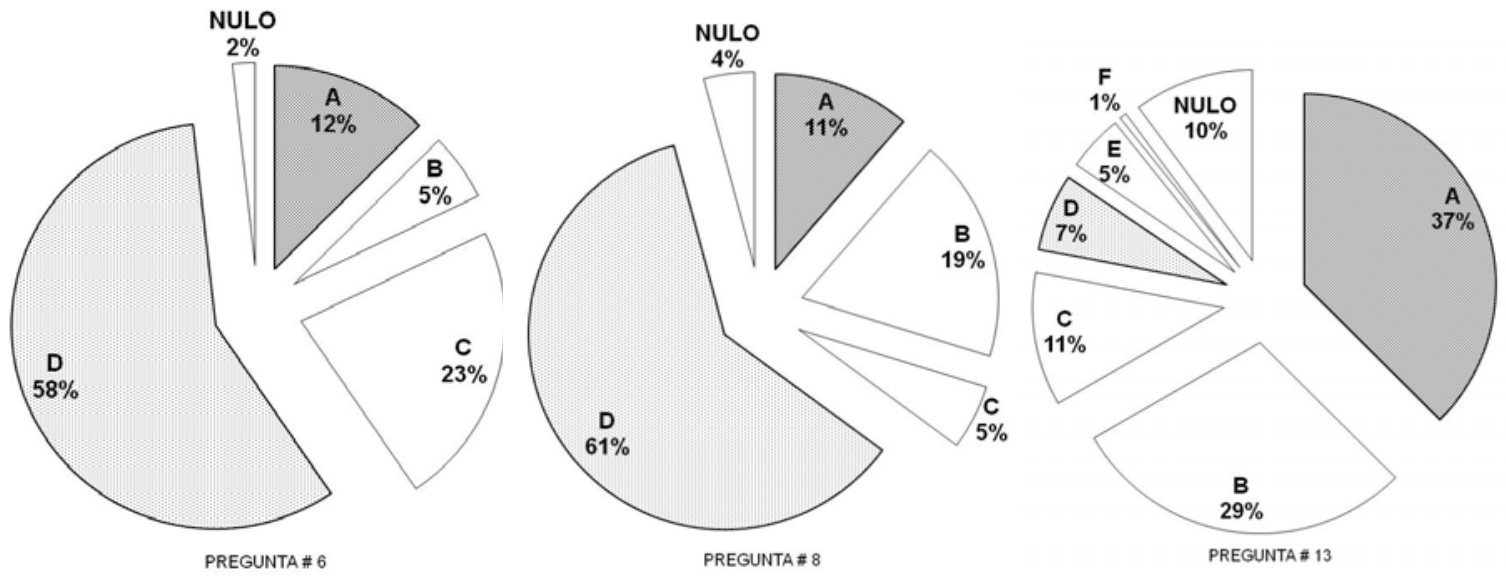

Figura 3. Conocimientos sobre manejo de complicaciones serias por calor. Pregunta \#6: La manera más efectiva y segura de enfriar a un atleta que se ha sobrecalentado y está sufriendo un golpe de calor es (A) sumergirlo en agua helada, (B) colocarle paños de alcohol en la frente, (C) colocarle hielo en las axilas y la ingle, (D) rociarlo repetidamente con agua fresca. Pregunta \#8: Cuando un triatlonista que está compitiendo en clima caliente y húmedo sufre un golpe de calor, lo primero que hay que hacer es (A) llamar una ambulancia y transportarlo al hospital más cercano, (B) darle suficiente líquido para que se hidrate bien, (C) acostarlo con las piernas elevadas, (D) enfriarlo primero y luego transportarlo al hospital. Pregunta \# 13: En una competencia en el calor, un atleta parece estar sufriendo de un golpe de calor. Para confirmar si tiene una hipertermia importante, el mejor método consiste en (A) tocar la frente con el dorso de la mano, (B) medir la temperatura axilar con un termómetro clínico, (C) medir la temperatura rectal con un termómetro clínico, (D) medir la temperatura sublingual con un termómetro clínico, (E) medir la temperatura timpánica con un dispositivo especial, (F) medir la temperatura intestinal con un termómetro ingerible.

\section{DISCUSIÓN.}

Los resultados de la encuesta son alarmantes, aunque no sorprenden, ya que la mayoría de los conocimientos medidos por ésta no son parte normal de los planes de estudio en Educación Física y Ciencias del Ejercicio (Clarkson, 2007). A continuación se procede a comentar los resultados de la encuesta y a profundizar en cada uno de los temas.

Tema 1: El riesgo de producir demasiado dolor (rabdomiólisis por esfuerzo). A pesar de ser un tema tan directamente relacionado con el profesional en Educación Física, un 93\% de los encuestados en el presente estudio afirmó (equivocadamente) que el dolor muscular retardado (DMR) es causado por la acumulación de ácido láctico en el músculo; solamente el $7.7 \%$ fueron capaces de identificar que los niveles altos de la enzima creatina fosfokinasa (CPK) en sangre pueden indicar que ha ocurrido rabdomiólisis; y aún menos, 3.6\% de los encuestados, indicaron que la mioglobina puede provocar una falla renal si su concentración en sangre se eleva mucho (ver Figura 1). Más de la mitad (51.8\%) indicaron que la mioglobina es la encargada de transportar el oxígeno en la sangre, cuando en realidad es la responsable de hacerlo dentro de las fibras musculares.

El dolor muscular retardado (DMR, o DOMS, por sus siglas en inglés) es un fenómeno bastante conocido por las personas físicamente activas. Se trata de un dolor muscular que aparece aproximadamente 24 horas después de haber realizado un ejercicio novedoso, y permanece durante 48 a 72 horas. El dolor va acompañado de una debilidad muscular que se puede medir fácilmente. Aún hoy día, algunas personas atribuyen este dolor a la presencia de ácido láctico en los músculos, sin embargo ya desde 1983 se había demostrado que el ácido láctico no tiene nada que ver con el DMR (Schwane, Johnson, Vandenakker \& Armstrong, 1983).

Hoy día es comúnmente aceptado en fisiología del ejercicio que este dolor obedece a microdesgarres que ocurren en las fibras musculares, y a la inflamación asociada con éstos. El DMR ocurre generalmente en personas que realizan algún tipo de ejercicio al que no están acostumbradas, especialmente ejercicio que involucra acciones excéntricas de los músculos, esto es, acciones en las cuales el músculo se extiende forzadamente mientras está activo. Los modelos de investigación más comunes para estudiar el DMR 
consisten en correr en una pendiente negativa (cuesta abajo), o realizar contracciones excéntricas de los músculos flexores del brazo. Es común que los profesionales en Educación Física vean el DMR como un resultado inevitable de iniciar un programa de acondicionamiento físico, pero la realidad es que si el programa se inicia progresivamente y se evitan las contracciones excéntricas en las primeras sesiones, el DMR puede ser mínimo o incluso estar totalmente ausente.

Probablemente por ser un fenómeno poco común, la complicación de un simple DMR en un caso más serio de rabdomiólisis por esfuerzo es casi desconocida. Este tipo de complicación, presentada magistralmente por la Dra. Priscilla Clarkson en la conferencia inaugural "Joseph B. Wolffe" durante el congreso anual del American College of Sports Medicine en Mayo del 2007, se define como la degeneración del músculo esquelético causada por ejercicio excesivo, no acostumbrado. Los síntomas de la rabdomiólisis incluyen dolor muscular, debilidad, e inflamación; mioglobinuria (presencia de mioglobina en la orina), y concentraciones elevadas de varias enzimas musculares en la sangre. La mioglobina en la orina le da una apariencia oscura, pero a veces la apariencia oscura obedece a una hemoglobinuria, una condición distinta que es mucho menos problemática y que se resuelve sola. A pesar de que estrictamente hablando todo DMR corresponde a grados moderados de rabdomiólisis (es decir, son lo mismo), clínicamente se ha definido la rabdomiólisis como aquella condición en la cual la concentración sanguínea de la enzima Creatina Kinasa (CPK) supera los 15,000 U/L; los niveles de $\mathrm{CPK}$ en reposo andan alrededor de $200 \mathrm{U} / \mathrm{L}$.

En algunos casos extremos, la mioglobina se puede precipitar en los riñones y causar una falla renal (Clarkson, 1993). Esto ha provocado la muerte de algunos individuos jóvenes y aparentemente sanos (Clarkson, 2006). Para prevenir esos pocos casos trágicos, es necesario que los profesionales en Educación Física estén conscientes de las posibles consecuencias serias de someter a los participantes de sus programas a rutinas extenuantes de ejercicios repetitivos y novedosos, tales como calistenia, ejercicios con pesas, o carrera, al inicio de un programa de acondicionamiento físico o a manera de castigo. También ayuda cuando el educador físico está atento a los participantes más agresivos y esforzados, que están voluntariamente más propensos a exagerar el ejercicio. Si algún participante nota que está produciendo orina oscura uno o dos días después de realizar una rutina de ejercicios novedosa y extenuante, debe consultar inmediatamente a su médico para descartar la posibilidad de mioglobinuria. Si se detecta mioglobinuria en un individuo, éste debe ser internado inmediatamente para observación y tratamiento.

Tema 2: La enfermedad de las células falciformes (ECF). Este síndrome pareciera ser poco conocido en Costa Rica. De hecho, de los participantes encuestados, solamente el 22\% reconoció que es peligroso utilizar el ejercicio como castigo o forzar a los estudiantes en el ejercicio, por existir casos de estudiantes que han muerto a consecuencia de esa práctica (sea por rabdomiólisis o por complicaciones asociadas con la ECF) (Clarkson, 2007).

Los glóbulos rojos, que son normalmente células en forma de disco achatadas en el centro como las desaparecidas pastillas costarricenses con sabor a menta o violeta marca Gallito, son un componente de la sangre y están encargadas de transportar el oxígeno a todas las células del cuerpo. La proteína en los glóbulos rojos, llamada hemoglobina, puede sufrir un trastorno leve de carácter hereditario que es normalmente inofensivo, la Enfermedad de Células Falciformes (ECF) o Drepanocitosis de Hemoglobina $\mathrm{S}$ en forma heterocigota (que no se debe confundir con su forma más grave, la drepanocitosis SS u homocigota). En condiciones de reposo, este trastorno pasa desapercibido. Sin embargo, durante el ejercicio de máxima intensidad, la alta demanda de oxígeno en los músculos puede hacer que los glóbulos rojos de las personas afectadas se transformen de su forma normal de disco a una forma de media luna u hoz, conocida como falciforme. Esto puede causar un bloqueo agudo en los vasos sanguíneos, provocando arritmias cardíacas o insuficiencia renal aguda. El resultado final puede ser la muerte.

En la población de los Estados Unidos de América, existe una prevalencia de este síndrome cercana a 1\% de la población. En personas de origen africano la prevalencia es mayor, casi del 9\%. Según datos reportados en el 2008 durante la sesión de declaratoria del 28 de Febrero como día nacional de lucha contra la drepanocitosis, se calcula que en Costa Rica la prevalencia de drepanocitosis heterocigota es de $10.9 \%$ en la población de raza 
negra, de $4.43 \%$ en la población mestiza, y de $0.82 \%$ en la población blanca. Puede visitar la siguiente página para mayor información http://www.fundrepa.org/principal/noticias/2008/dia nacional.html

La mayoría de estas personas viven vidas normales, pero especialmente los atletas, debido a que a menudo se exponen a esfuerzos máximos, tienen un riesgo importante de sufrir serios problemas y hasta de morir. Como ejemplo, está documentado que el riesgo de muerte súbita entre reclutas del ejército de los Estados Unidos es 40 veces mayor en aquéllos con síndrome de células falciformes, que en todos los demás reclutas (Kark, Posey, Schumacher \& Ruehle, 1987).

En el síndrome de células falciformes, durante el ejercicio de máxima intensidad, existen cuatro fuerzas o factores que se combinan para producir el ambiente propicio para la deformación o polimerización de los glóbulos rojos: las primeras dos son la acidosis y la elevación en la temperatura del tejido muscular, que producen un desplazamiento de la curva de asociación/disociación del oxígeno y la hemoglobina hacia la derecha, fomentando la disociación. La tercera es la deshidratación de los glóbulos rojos, que aumenta la concentración de hemoglobina en ellos. Y la cuarta es que hay una altísima demanda de oxígeno por parte de los músculos. El resultado es una concentración extremadamente baja de oxígeno en la hemoglobina, que precipita el problema.

El riesgo de producir células falciformes aumenta directamente con la dificultad del ejercicio, de manera que cualquier aspecto que haga que el ejercicio sea más difícil, como la deshidratación, el calor ambiental, la altitud y el asma, va a incrementar la posibilidad de deformación de los glóbulos rojos. Obviamente, el tipo de entrenamiento y la presión que exista sobre el atleta de dar su máximo esfuerzo, inciden directamente en la dificultad del ejercicio: pareciera que cuanto más rápido vaya el atleta, tanto más rápida y difundida será la transformación de los glóbulos rojos. ¿Es necesario evitar el esfuerzo máximo en todos los deportistas? Realmente no, sino solamente en aquéllos que por tener un diagnóstico de ECF o alguna otra limitación médica, deban moderar su esfuerzo.

Uno de los problemas con el colapso por ECF es que sus síntomas son a menudo confundidos con los calambres por calor, el agotamiento por calor, y el golpe de calor. Al no darse oportunamente el tratamiento indicado, la condición se deteriora rápidamente. Por ésta y otras razones, lo mejor es la prevención para evitar que los deportistas con ECF provoquen la transformación de sus glóbulos rojos.

El primer paso en la prevención de complicaciones por ECF es el diagnóstico oportuno. Esto es relativamente sencillo, basta con un examen de sangre para detectar hemoglobinopatías. Este tipo de examen aún no se hace oficialmente como tamizaje en Costa Rica, pero se puede solicitar expresamente en el CIHATA (Centro de Investigaciones en Hemoglobina y Trastornos Afines de la Universidad de Costa Rica), pidiendo una Electroforesis de Hemoglobina. Una vez que el deportista y su entrenador saben de la presencia de ECF, se pueden evitar los esfuerzos de máxima intensidad tales como piques de velocidad repetidos, "parrillas" o carrera suicida, y el entrenamiento máximo en altitud, en el calor, o en condiciones de deshidratación. Cualquier calambre, dificultad o colapso en un deportista con ECF debe considerarse como una complicación de su enfermedad, que sería una emergencia médica, hasta que se demuestre lo contrario. Se recomienda revisar la publicación de la referencia del Dr. Eichner (2006) para recomendaciones más completas sobre este tema. Para información en Costa Rica, aunque el tema específico asociado al deporte no se menciona, se puede visitar la página de la Fundación para la Investigación y Apoyo al Paciente con Drepanocitosis/ Hemoglobinopatías, en http://www.fundrepa.com/principal/hemo.htm

Tema 3: El riesgo de que nos parta un rayo. A la pregunta sobre cómo proceder cuando hay una tormenta eléctrica a cinco kilómetros de una cancha abierta, alrededor de la mitad (47.6\%) sugirió suspender las actividades y buscar refugio, esperando 30 minutos sin caída de rayos antes de reiniciar; $31.5 \%$ de los encuestados subestimó el número anual de muertos por rayería en Costa Rica, aunque el $40.5 \%$ sí acertó indicando que es de alrededor de 7 personas por año (Figura 2).

Asterix y sus valientes amigos galos temían solamente una cosa: que cayera el cielo sobre sus cabezas. Quizás estaban muy conscientes del peligro de los rayos. Sobre este tema se bromea mucho, y hay quienes dicen que la probabilidad de que a uno lo parta un rayo es demasiado baja como para preocuparse. Cierto, a menos que uno esté en el 
lugar perfecto en el momento equivocado. Por ejemplo, según el periódico La Nación (Arguedas, 2006), en Costa Rica cae un promedio de 1100 (mil cien) rayos por día, lo cual provocó 15 muertes en dos años. Algunas de esas muertes, como la de Jorge Alvarado Varela de 32 años, ocurren mientras la gente juega fútbol (Vargas \& Hernández, 2005).

La seriedad del problema motivó a la Asociación Nacional de Kinesiólogos de los Estados Unidos (NATA, siglas en inglés de National Athletic Trainers' Association) a declarar su posición oficial. (Walsh, Bennett, Cooper, Holle, Kithil \& López, 2000).

En esta declaración se informa que cada año mueren casi 100 personas y 400 sufren lesiones como consecuencia de la rayería en su país, y que una proporción alarmante de esos accidentes involucran personas que estaban participando en eventos deportivos o recreativos al aire libre. Una de las recomendaciones más importantes de la NATA es que los profesionales involucrados en las actividades deportivas y recreativas deben estar debidamente informados acerca de los peligros de los rayos, y acerca de las precauciones que se deben tomar al respecto.

Quizás una de las precauciones más importantes y fáciles de aplicar consiste en suspender la práctica deportiva al aire libre y buscar refugio apropiado cuando hay una tormenta eléctrica en el vecindario. La NATA recomienda suspender actividades cuando transcurren 30 segundos o menos entre la luz del rayo y el trueno correspondiente. Esto podría considerarse un poco exagerado, ya que una diferencia de 30 segundos representa una distancia de 10 kilómetros, pero en cosas así a veces es mejor ser demasiado conservador que demasiado arriesgado.

Para calcular la distancia a que se encuentra una tormenta, se deben contar o cronometrar los segundos que transcurren entre el instante en que se observa la caída de un rayo y el momento en que se escucha el trueno. Debido a la diferencia de velocidad de la luz y el sonido (300 $000 \mathrm{~km} / \mathrm{s}$ y 330 $\mathrm{m} / \mathrm{s}$, respectivamente), se puede calcular la distancia en kilómetros dividiendo los segundos transcurridos entre ambos eventos por 3. Eso indica la distancia a la que cayó ese rayo, aunque la tormenta podría estar más cerca ya que las tormentas son grandes. Pero al menos se tiene una idea aproximada.

El conteo de segundos entre rayos y truenos también permite calcular si la tormenta se acerca o se aleja, según el cambio de la diferencia de tiempo de un rayo al siguiente: cuando la tormenta se acerca, el tiempo disminuye; cuando se aleja, éste aumenta. En todo caso, lo importante es que las actividades al aire libre se deben suspender cuando hay rayería cerca, se deben evitar los espacios abiertos, y no se debe buscar refugio bajo los árboles. Se debe suspender toda actividad de natación, sea bajo techo o al aire libre. No se deben utilizar los teléfonos convencionales, pues la corriente de los rayos se transmite por las líneas telefónicas. Los electrodomésticos y las duchas, por estar conectados a la tubería, pueden también transmitir la electricidad. Los refugios seguros son cualquier casa o edificio cerrado o, en casos extremos, un automóvil con las puertas y ventanas cerradas, evitando estar en contacto con las partes metálicas. La NATA recomienda esperar 30 minutos después del último trueno, antes de reiniciar las actividades al aire libre.

Tema 4: El golpe de calor por esfuerzo. ¿Cómo enfriar rápidamente a un atleta sobrecalentado? Es satisfactorio observar que el $60.7 \%$ de los encuestados fue capaz de identificar que si un triatlonista sufre de un golpe de calor, lo primero que hay que hacer es enfriarlo, y luego transportarlo al hospital. Sin embargo, es altamente preocupante que los profesionales en educación física aparentemente no saben cómo medir la temperatura corporal: $37.5 \%$ indicaron que el mejor método para confirmar si un atleta tiene un grado importante de hipertermia es tocar la frente con el dorso de la mano, y 29.2\% que debe medirse la temperatura axilar con un termómetro clínico; solamente $11.3 \%$ supieron identificar la medición de la temperatura rectal con termómetro clínico como el mejor método (Figura 3). También es evidente que los encuestados no saben cuál es la manera más efectiva y segura de enfriar a un atleta que se ha sobrecalentado y está sufriendo un golpe de calor: $57.7 \%$ lo rociarían repetidamente con agua fresca, y 22.6\% le colocarían hielo en las axilas y la ingle, procedimientos que se ha demostrado que tienen coeficientes de enfriamiento de sólo $0.15 \mathrm{y}$ $0.03{ }^{\circ} \mathrm{C}$ por minuto, respectivamente. En comparación, la inmersión en agua helada tiene un coeficiente de $0.35^{\circ} \mathrm{C} / \mathrm{min}$, el mejor coeficiente de enfriamiento (Casa, et al., 2007) que fue escogida por solamente $12.5 \%$ de las personas encuestadas (Figura 3). 
Ocasionalmente, cuando una competición o entrenamiento se lleva a cabo en condiciones de alta temperatura y humedad, algunos de los participantes se pueden sobrecalentar a tal punto que llegan a sufrir de un golpe de calor, una condición potencialmente letal en que los mecanismos para regular la temperatura corporal dejan de funcionar y la temperatura central se eleva sin control, provocando daños a uno o más órganos. El riesgo siempre existe cuando las condiciones ambientales, la intensidad y duración del esfuerzo, la deshidratación y el tipo de vestimenta favorecen la alta producción de calor metabólico y limitan la liberación de ese calor. Por eso es que las medidas más importantes para prevenir complicaciones por calor incluyen el monitoreo de las condiciones ambientales, la modificación de los entrenamientos cuando hay mucho estrés por calor, el uso de ropa clara y liviana, y la buena hidratación. Recientemente, se ha recomendado el prestar atención especial a los individuos que podrían estar particularmente predispuestos a tener problemas, a saber, aquéllos que inician el entrenamiento ya deshidratados, los que tienen un índice de masa corporal superior a $22 \mathrm{~kg} * \mathrm{~m}^{-2}$, y los que están en mala condición física o poco aclimatados a ejercitarse en el calor (Cleary, 2007).

Ahora bien, cuando a pesar de las medidas preventivas (o debido a su omisión) sucede que una o más personas sufren un golpe de calor por esfuerzo, es esencial proceder de la manera apropiada lo más rápido posible. En la declaración oficial reciente del Colegio Americano de Medicina Deportiva (ACSM, siglas en Inglés de American College of Sports Medicine). (Armstrong, Casa, Millard-Stafford, Moran, Pyne, \& Roberts, 2007) se define el golpe de calor por esfuerzo como un aumento en la temperatura central del cuerpo por arriba de los $40^{\circ} \mathrm{C}$, asociada con perturbaciones del sistema nervioso central y la falla de múltiples órganos. Cuando se sospecha de un caso de golpe de calor por esfuerzo, los dos pasos cruciales son a) la medición de la temperatura corporal central, y b) el enfriamiento rápido. La única medición confiable de la temperatura central en estas circunstancias es la temperatura rectal. Existen dispositivos de uso clínico frecuente, tales como el termómetro timpánico o el rastreador (scanner) temporal, que son más prácticos, pero se ha demostrado experimentalmente que sus resultados no son válidos en casos de golpe de calor por esfuerzo, es decir, no detectan los aumentos de temperatura central que en realidad están ocurriendo (Casa, Armstrong, Ganio, \& Yeargin, 2005; Low, Vu, Brown, Davis, Keller, Levine, \& Crandall, 2007). El uso de termómetros ingeribles para telemetría es factible, pero su practicalidad es limitada pues tendría que darse la casualidad de que el atleta que se debe medir hubiera ingerido una cápsula varias horas antes.

$\mathrm{Si}$ se encuentra que el atleta tiene una temperatura rectal superior a los $40^{\circ} \mathrm{C}$, el enfriamiento rápido es crucial. La evidencia muestra claramente que la severidad de las lesiones que ocurren durante un golpe de calor por esfuerzo, depende directamente del tiempo que la temperatura rectal se mantiene por encima del umbral de daños celulares. El objetivo primario del tratamiento en el golpe de calor debe ser bajar la temperatura central por debajo de $40^{\circ} \mathrm{C}$ en menos de 30 minutos; el enfriamiento se debe detener al llegar a una temperatura central de $39^{\circ} \mathrm{C}$, ya que de continuar se puede más bien provocar una hipotermia, facilitada por la inercia de la temperatura central y por el mal funcionamiento de la termorregulación. Para el enfriamiento, el mejor método es la inmersión de todo el cuerpo, excepto la cabeza, en agua fría o helada, siempre que las condiciones particulares del atleta lo permitan; este método es efectivo y tiene las tasas de enfriamiento corporal más altas demostradas experimentalmente (Casa, McDermott, Lee, Yeargin, Armstrong, \& Maresh, 2007). Otras alternativas menos efectivas incluyen el baño profuso con agua fría, o la aplicación de paños con agua de hielo o hielo. La selección del mejor método también depende de qué tan pronto se puede empezar su aplicación. Lo importante es recordar que la inmersión en agua fría o agua helada es el método más efectivo, sencillo y rápido para bajar la temperatura central de personas con golpe de calor por esfuerzo. Este enfriamiento debe iniciarse lo antes posible (la regla es enfriar primero, trasladar después).

En conclusión, este estudio ha confirmado que los estudiantes y profesionales en Educación Física en Costa Rica no están debidamente informados sobre la prevención y el manejo de cuatro asuntos de vida o muerte relacionados directamente con su profesión, a saber: la rabdomiólisis por esfuerzo, el síndrome de células falciformes, el peligro de accidentes por descargas eléctricas atmosféricas, y las complicaciones del golpe de calor por esfuerzo. 
La información presentada en este artículo debe ser incorporada a los contenidos de los planes de estudio para Educadores Físicos, Entrenadores Personales, y otros profesionales de las Ciencias del Ejercicio, de manera que éstos puedan mejorar la seguridad y la experiencia de las personas a su cargo. También se evidencia la importancia de comunicar esta información a tantos profesionales como sea posible, en el contexto de los programas de educación continua o actualización profesional.

\section{REFERENCIAS}

Arguedas, C. (2006, Noviembre 5). 1.100 rayos caen por día como promedio en el país. $L a$ Nación. Recuperado el 03 de Septiembre de 2007, desde http://www.nacion.com/ln_ee/2006/noviembre/0 5/pais876241.html

Armstrong, L. E., Casa, D. J., Millard-Stafford, M., Moran, D. S., Pyne, S. W., \& Roberts, W. O. (2007). American College of Sports Medicine position stand. Exertional heat illness during training and competition. Medicine \& Science in Sports \& Exercise, 39(3), 556-572.

Casa, D. J., Armstrong, L. E., Ganio, M. S., \& Yeargin, S. W. (2005). Exertional heat stroke in competitive athletes. Current Sports Medicine Reports, 4(6), 309-317.

Casa, D. J., McDermott, B. P., Lee, E. C., Yeargin, S. W., Armstrong, L. E., \& Maresh, C. M. (2007). Cold water immersion: the gold standard for exertional heatstroke treatment. Exercise and Sport Sciences Reviews, 35(3), 141-149.

Clarkson, P. M. (1993). Worst Case Scenarios: Exertional Rhabdomyolysis and acute renal failure. Sports Science Exchange, 4(42), 1-6.

Clarkson, P. M. (2006). Case report of exertional rhabdomyolysis in a 12-year-old boy. Medicine \& Science in Sports \& Exercise, 38(2), 197-200.

Clarkson, P.M (2007, mayo). Joseph B. Wolffe. Conferencia Inaugural presentada en el Congreso Anual del American College of Sports Medicine. Saint Louis, Missouri.
Cleary, M. D (2007). Predisposing Risk Factors on Susceptibility to Exertional Heat Illness: Clinical Decision-Making Considerations. Journal of Sport Rehabilitation, 16(3), 204-214.

Eichner, E. R. (2006). Enfermedad de células falciformes y el atleta. Sports Science Exchange, 19(4), 1-6.

Kark, J. A., Posey, D. M., Schumacher, H. R., \& Ruehle, C. J. (1987). Sickle-cell trait as a risk factor for sudden death in physical training. New England Journal of Medicine, 317(13), 781-787.

Low, D. A., Vu, A., Brown, M., Davis, S. L., Keller, D. M., Levine, B. D., \& Crandall, C. G. (2007). Temporal thermometry fails to track body core temperature during heat stress. Medicine \& Science in Sports \& Exercise, 39(7), 1029-1035.

Ronneberg, K., Roberts, W. O., McBean, A. D., \& Center, B. A. (2008). Temporal artery temperature measurements do not detect hyperthermic marathon runners. Medicine \& Science in Sports \& Exercise, 40(8), 1373-1375.

Schwane, J. A., Johnson, S. R., Vandenakker, C. B., \& Armstrong, R. B. (1983). Delayed-onset muscular soreness and plasma CPK and LDH activities after downhill running. Medicine \& Science in Sports \& Exercise, 15(1), 51-56.

Vargas, O., y Hernández, C. (2005, Noviembre 3). Rayos mataron a 12 personas en los últimos ocho meses. La Nación. Recuperado el 03 de Septiembre de 2007, desde http://www.nacion.com/ln_ee/2005/noviembre/0 3/sucesos1.html

Walsh, K.M., Bennett, B., Cooper, M.A., Holle, R.L., Kithil, R., \& López, R.E. (2000). National Athletic Trainers' Association Position Statement: Lightning Safety for Athletics and Recreation. Journal of Athletic Training, 35(4), 471-477. 


\title{
ANEXO 1: ENCUESTA
}

\author{
Universidad de Costa Rica
}

Escuela de Educación Física y Deportes

\section{Encuesta sobre conceptos aprendidos en la carrera.}

Bienvenidos al XIV Simposio de Ciencias del Ejercicio y la Salud de la Escuela de Educación Física de la Universidad de Costa Rica. En esta oportunidad, hemos querido realizar una pequeña encuesta para evaluar, en forma anónima, qué tan bien recuerdan los profesionales en Educación Física algunos de los conceptos que aprendieron durante sus estudios universitarios o que se han discutido en ediciones anteriores del simposio. Le agradecemos que tome unos minutos para contestar cuidadosamente las siguientes preguntas.

Atentamente,

Luis Fernando Aragón V., Ph.D., FACSM

MARQUE CON UNA X LA MEJOR OPCION: Por favor, conteste a conciencia.

1. El arratonamiento (dolor muscular retardado) se presenta frecuentemente en personas que no están acostumbradas al ejercicio y limita la actividad física por unos días. Diría Ud. que esta complicación por el ejercicio...

a) Es algo pasajero que siempre se resuelve solo, con el tiempo

b) Es potencialmente mortal

c) Es causada por la acumulación de ácido láctico en el músculo

d) Es provocada por contracciones concéntricas

2. La única sustancia que se puede utilizar como combustible en condiciones anaeróbicas es:

a) La nicotina

b) El ácido linolénico

c) La glucosa

d) Los ácidos grasos libres

3. Algunos doctores se alarman cuando una persona tiene niveles elevados de CPK en sangre, ya que eso es un indicador de posible lesión en el músculo cardiaco (podría indicar que hubo un infarto al miocardio). ¿En qué otras condiciones podría elevarse el CPK?

a) Después de nadar en agua muy fría

b) Cuando hay acidosis excesiva durante el ejercicio intenso

c) Cuando ha ocurrido rabdomiólisis

d) Por deshidratación aguda 
4. Cuando hay una tormenta eléctrica a cinco kilómetros de una cancha abierta de fútbol, es necesario suspender la mejenga (juego colectivo) o entrenamiento y buscar un refugio adecuado

a) ... y esperar 30 minutos después de la caída del último rayo

b) sólo si los rayos tienen una frecuencia muy alta (más de 2 rayos por minuto)

c) sólo si parece que la tormenta se acerca

d) No es necesario suspender la mejenga

5. En la escuela y el colegio, el educador físico nunca debe utilizar el ejercicio como castigo porque

a) La Sala IV acogió un recurso de amparo que impide hacerlo

b) Los alumnos pueden desarrollar una actitud negativa hacia el ejercicio

c) Existen casos de estudiantes que han muerto por esta causa

d) Las respuestas b y c son correctas

6. La manera más efectiva y segura de enfriar a un atleta que se ha sobrecalentado y está sufriendo un golpe de calor es

a) Sumergirlo en agua helada

b) Colocarle paños de alcohol en la frente

c) Colocarle hielo en las axilas y la ingle

d) Rociarlo repetidamente con agua fresca

\section{Para la práctica de la natación, se deben observar siempre las siguientes reglas básicas:}

a) Evitar zonas cerca de la playa donde hay corrientes de resaca

b) Nunca nadar solo

c) No nadar cuando hay rayería cerca

d) Todas las anteriores son correctas

8. Cuando un triatlonista que está compitiendo en clima caliente y húmedo sufre un golpe de calor, lo primero que hay que hacer es

a) Llamar a una ambulancia y transportarlo al hospital más cercano

b) Darle suficiente líquido frío para que se hidrate bien

c) Acostarlo con las piernas elevadas

d) Enfriarlo primero, y luego transportarlo al hospital

9. Cuando de hidratación se trata, hay que recordar que

a) Todas las personas deben beber un mínimo de 8 vasos diarios de agua

b) Es imposible beber demasiada agua, pues los riñones siempre eliminan el exceso

c) Las personas sedentarias se hidratan suficientemente bien con sólo obedecer el mecanismo de la sed

d) Toda las bebidas son iguales, lo que cuenta es el líquido 
10. Una sustancia muy importante para la función muscular es la mioglobina. Esta sustancia

a) Transporta el oxígeno en la sangre

b) Puede provocar una falla renal si su concentración en sangre sube mucho

c) Está relacionada con la potencia y capacidad anaeróbica muscular

d) Es una hormona regulada por el metabolismo aeróbico

11. La creatina es un suplemento nutricional ampliamente utilizado por los deportistas. Existe evidencia experimental clara de que la creatina

a) Podría provocar daños musculares si se ingiere en exceso

b) Mejora significativamente el rendimiento deportivo en atletismo de fondo

c) Provoca deshidratación y calambres musculares

d) Mejora el tiempo en pruebas de velocidad (100, 200, 400m) en un 30\%

12. En Costa Rica muere un número importante de personas por descargas eléctricas (rayería) al año mientras trabajan, se recrean, o practican deporte al aire libre, a saber

a) 2 personas por año

b) 4 personas por año

c) 7 personas por año

d) 20 personas por año

13. En un entrenamiento o competencia en el calor, un atleta se agota y pareciera estar sufriendo un golpe de calor. En ese momento, para confirmar si tiene una hipertermia importante, el mejor método consiste en

a) Tocar la frente con el dorso de la mano

b) Medir la temperatura axilar con un termómetro clínico

c) Medir la temperatura rectal con un termómetro clínico

d) Medir la temperatura sublingual con un termómetro clínico

e) Medir la temperatura timpánica con un dispositivo especial

f) Medir la temperatura intestinal con un termómetro ingerible

14. Cuando un(a) estudiante sufre una torcedura de tobillo en clase de educación física, hay dolor y rápidamente comienza la inflamación, el profesor debe

a) Aplicar hielo e inmovilizar, antes que nada

b) Trasladar inmediatamente al hospital para evaluación

c) Aplicar calor y masaje

d) Dar RCP

Gracias por su colaboración. 